\title{
The residual deprivation effect in instrumental conditioning as a function of deprivation level at the time of exposure to the reinforcer
}

\author{
DANE PETERSON and JAMES H. McHOSE \\ Southern Illinois University at Carbondale, Carbondale, Illinois 62901
}

\begin{abstract}
The effect of food deprivation level at the time of initial exposure to a subsequent food reinforcer was investigated in two experiments. In Experiment 1, deprivation at the time of initial exposure influenced the subsequent acquisition and extinction of an instrumental response. In Experiment 2, the residual deprivation effect associated with a reduction in deprivation level occurred only when rats initially experienced the reinforcer at a high, as compared with a low, deprivation level. Results were discussed in terms of the assumption that the limits of incentive generated by a reinforcer are influenced by the deprivation state at the time of first exposure to that reinforcer.
\end{abstract}

It is well known that the prevailing level of food deprivation influences responding in simple appetitive instrumental conditioning, a fact that is typically attributed to the effects of privation on motivation (Hull, 1943). Also, under certain conditions, the deprivation level at the time of response acquisition affects subsequent performance levels. Several investigations (Capaldi, 1971, 1972a; Capaldi \& Hovancik, 1973; Zaretsky, 1966) have shown that subjects trained under high deprivation and then shifted to a lower level of food deprivation continue to run faster in a straight alley for a food reward than do subjects trained and maintained at the lower level of deprivation. The present studies were concerned with an interpretation of this residual deprivation effect.

Given satisfactory precautions to insure that subjects originally trained on high deprivation are indeed at low deprivation during the postshift period of a deprivation shift experiment, the residual deprivation effect is not interpretable with the simple assumption that deprivation affects motivational states. For this reason, interpretations of the residual deprivation effect have typically assumed that deprivation influences the associative strength formed between the stimulus and the instrumental response (habit strength). Several writers (Capaldi, 1972b; Hovancik, 1978; Zaretsky, 1966) have proposed that greater habit strength accrues when a response is rewarded under a high, as compared with a lower, level of deprivation.

An alternative solution would be to assume that deprivation affects the incentive value of the reward,

\footnotetext{
This research is based on a thesis submitted by Dane K. Peterson, under the direction of James H. McHose, to the Graduate School, Southern Illinois University at Carbondale, in partial fulfillment of requirements for the MA degree. Requests for reprints should be sent to James H. McHose, Department of Psychology, Southern Illinois University at Carbondale, Carbondale, Illinois 62901.
}

a notion suggested by numerous investigators (Bindra, 1972; Kurtz \& Jarka, 1968; Mollenauer, 1971; Revusky, 1968). According to this view, the incentive value of a nutrient substance is assumed to vary as a function of the deprivation state of the organism: the higher the deprivation state, the higher the resulting incentive value. Because both hypotheses assume that deprivation influences a relatively permanent mechanism (incentive or habit) that does not change immediately when deprivation level is shifted, both are capable of providing an explanation of the residual deprivation effect.

In agreement with the assumption that deprivation states influence incentive levels, Revusky (1967) found that rats fed one solution while hungry and another while nearly satiated subsequently preferred the solution consumed while hungry. Analogous effects, persisting through 10 days of preference testing, have been observed under thirst states (Revusky, 1974). Kurtz and Jarka (1968) also found increased preference for a choice response rewarded while hungry over a response rewarded while nearly satiated.

While previous taste preference and choice data suggest a relationship between the reward or incentive value of a substance and deprivation history, these paradigms are not directly applicable to the betweensubjects designs and response measures of the deprivation shift studies. Experiment 1 was designed to determine whether variations in the deprivation level at the time of initial exposure to a food reward would affect subsequent instrumental responding in a manner similar to variation in reward magnitude, a variable generally assumed to influence incentive level (Spence, 1956). Specifically, Experiment 1 sought to determine whether exposure to reinforcement pellets under high deprivation, as compared with low depri- 
vation, would affect subsequent instrumental performance under low deprivation. If deprivation state at the time of exposure to the reinforcer were to affect instrumental performance in ways analogous to reward magnitude manipulations, two effects of the deprivation variable should be apparent. First, highdeprivation exposure should produce heightened acquisition performance in intermediate stages of acquisition (cf. McHose \& Moore, 1976b). Second, high-deprivation exposure should reduce resistance to experimental extinction (cf. Mackintosh, 1974, pp. 427-428).

Although there appears to be no direct evidence concerning the effect of novelty of food substance on the taste preference phenomena reported by Revusky $(1967,1968,1974)$, conditioned taste aversion phenomena are clearly best observed with novel food substances (Best, 1975; Kalat, 1974). The implication of these data, as well as the theoretical relationship of aversion to taste preference data (Revusky \& Garcia, 1970), in the present context would appear to be that augmenting the incentive level of reinforcement pellets by exposure to these pellets under high deprivation would be best accomplished when a subject's initial exposure to the pellets is under high deprivation. Experiment 1 addressed the possibility.

\section{EXPERIMENT 1}

\section{Method}

Subjects. The subjects were 28 experimentally naive, 180-dayold, male rats obtained from the Holizman Company, Madison, Wisconsin.

Apparatus. The apparatus was a straight alley, painted flat black and covered by a translucent Plexiglas ceiling, with a 25 $\mathrm{cm}$-long startbox, an $80-\mathrm{cm}$ run section, and a $25-\mathrm{cm}$ goal section. The inner width and height of each section were $9 \mathrm{~cm}$. A side-exiting, solenoid-operated, opaque startdoor separated the startbox from the running section, and a translucent guillotinetype retrace door separated the run and goal sections. Photocellclock circuitry provided traversal times over the first three $30-\mathrm{cm}$ segments of the alley. A Panmotor Model 1000A fan mounted on a rear wall of the goalbox extracted air from the apparatus via $30-\mathrm{mm}$ holes in the rear wall.

Design. A 2 by 2 factorial design was employed with three stages of training. The four groups were labeled according to the variables in the factorial design: the deprivation state of the subjects during the flavor conditioning phase (second phase) and whether the reward was a familiar flavor or a novel flavor at the time of flavor conditioning. The four groups were HiD.F (high deprivation, familiar flavor), LoD-F, HiD-N, and LoD-N. Seven subjects were randomly assigned to each group.

The purpose of the first stage was to familiarize Groups HiD-F and LoD-F with the test flavor (Noyes food pellets). In the second, or flavor conditioning, phase, subjects in Groups HiD-F and HiD-N were allowed to consume the test flavor only under high deprivation, while subjects in groups LoD-F and LoD-N were allowed to consume the same flavor only under low deprivation. In the final stage, all subjects were tested under low deprivation in the straight alley.

Procedure. Twelve days prior to the start of the flavor conditioning phase, all subjects were placed on a 23-h food deprivation cycle that was maintained throughout the experiment. Cage feeding consisted of $1 \mathrm{~h}$ of free access to Purina Rat Chow.
One hour after feeding, subjects in Groups HiD-F and LoD-F were allowed to consume 22.045 -g Noyes food pellets per day in their home cage.

During the flavor conditioning phase, subjects in Groups HiD-F and HiD-N were allowed to consume 22 pellets $21 \mathrm{~h}$ after cage feeding each day, while the subjects in Groups LoD-F and LoD-N were allowed to consume 22 pellets 1 to $2 \mathrm{~h}$ after cage feeding. The flavor conditioning phase lasted for 10 days, during which time all subjects were handled for about $5 \mathrm{~min}$ a day. During the last 4 days, subjects were allowed to explore the test apparatus.

In the test, or runway training, phase, all subjects received runway training 1 to $2 \mathrm{~h}$ after their daily cage feeding (i.e., all subjects were trained under low deprivation). All subjects received five reinforced trials per day for 5 days. Following acquisition training, all groups were extinguished over the next 3 days at five trials per day. The procedure was the same as it was in acquisition, except that the subjects were confined to the goalbox without reinforcement for $20 \mathrm{sec}$.

Subjects were run in squads of seven with no more than two subjects from the same group in any squad. The resulting intertrial interval was approximately $8 \mathrm{~min}$. A running trial was initiated by placing the subject in the startbox. Following a $3-\mathrm{sec}$ orientation to the startdoor, the animal was allowed to traverse the runway. Upon entering the goalbox, the retrace door was lowered. Subjects were removed from the goalbox immediately after consumption of the reward.

\section{Results}

Since the various response measures yielded similar information, only the reciprocal total alley latencies will be presented. Group mean total speeds are plotted in blocks of five trials in Figure 1. As may be seen in Figure 1, subjects that were allowed to consume the pellets only under high deprivation in the flavor conditioning phase ran faster through intermediate stages of acquisition than did subjects that received the pellets under low deprivation. Analyses of variance yielded a significant deprivation effect over Days 3 and 4 combined $[F(1,24)=4.57, p<.05]$, but not on Days 1 and 2 or on Day 5 (Fs $<1$ ).

As Figure 1 shows, only Group HiD-N was less resistant to extinction than its control (Group LoD-N). Analysis of variance of the terminal acquisition (Block 5) and terminal extinction (Block 8) data yielded a significant Deprivation by Blocks by Familiarity interaction $[F(1,24)=5.59, \mathrm{p}<.05]$. Subsequent analyses yielded a significant Blocks by Deprivation interaction for subjects in the novel condition only $[F(1,24)=8.11, p<.01]$ and a significant Deprivation by Familiarity interaction at Block 8 only $[F(1,24)=$ $9.69, \mathrm{p}<.01]$. At Block 8, Group HiD-N ran more slowly than Group LoD-N $[\mathrm{F}(1,24)=11.60, \mathrm{p}<.01]$, while no differences were obtained between Groups HiD-F and LoD-F $(F<1)$.

\section{Discussion}

In Experiment 1, the acquisition and extinction of an instrumental response under a low deprivation state were influenced by the deprivation state that was in effect when subjects were exposed to the reinforcer prior to instrumental conditioning. Group HiD-N, which experienced the reinforcer for the first 


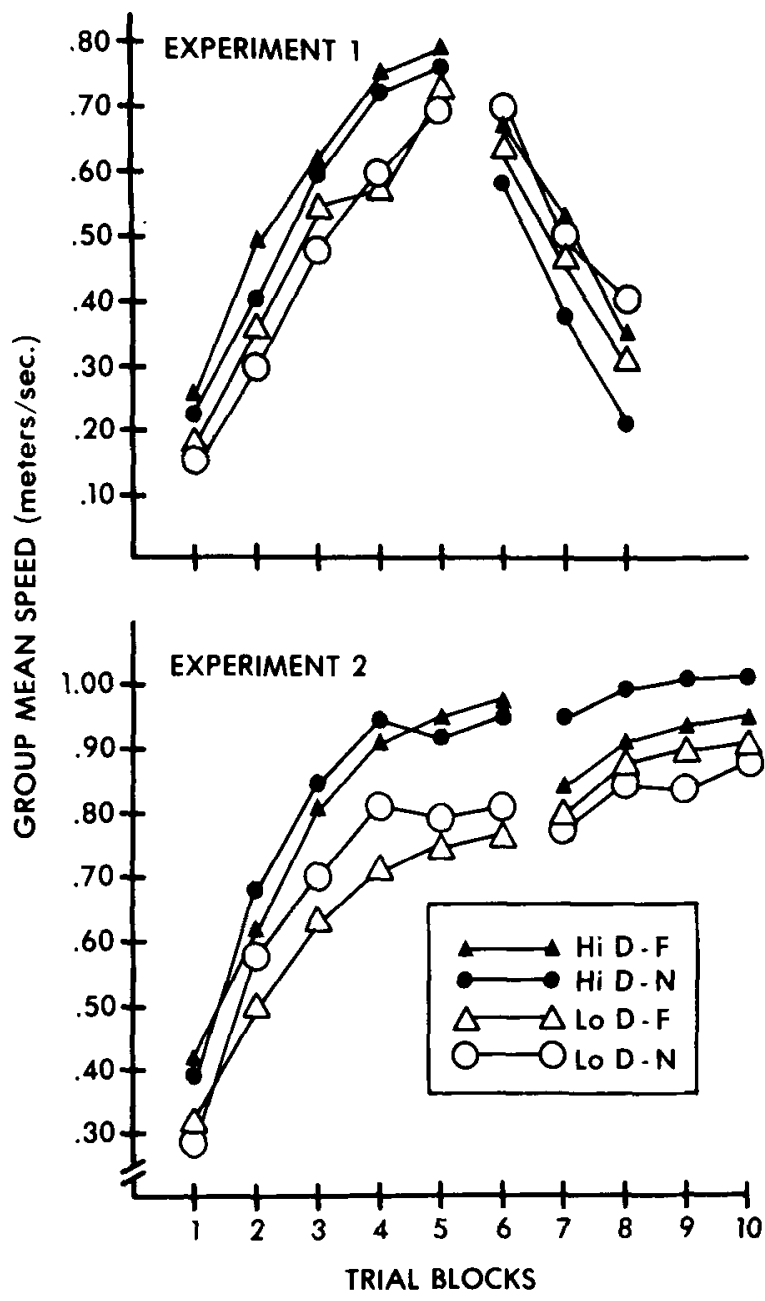

Figure 1. Group mean speeds as a function of blocks of five trials for Experiments 1 and 2. Group identifications (lower panel) apply to both experiments.

time under high deprivation, ran faster through intermediate stages of acquisition and extinguished more rapidly than did Group LoD-N. The relative performance levels of Groups HiD-N and LoD-N during both acquisition and extinction are directly analogous to comparisons between large- and small-rewardmagnitude groups in acquisition (McHose \& Moore, 1976b) and extinction (Mackintosh, 1974, pp. 427-428). For theoretical frameworks employing an incentive concept, reward magnitude is generally assumed to alter incentive (cf. McHose \& Moore, 1976a; Spence, 1956). Thus, the observation that deprivation influences performance in a manner similar to variation in reward magnitude is consistent with the notion that deprivation states affect the incentive value of reinforcer substances.

It was anticipated that exposure to the reinforcer under high deprivation would augment incentive levels only if subjects were originally exposed to the reinforcer under high deprivation. The extinction data, in which only the novel reward groups differed, are consistent with this expectation. The acquisition data are not consistent, because both Groups HiD-N and HiD-F displayed heightened performance levels relative to Groups LoD-N and LoD-F.

\section{EXPERIMENT 2}

The results of Experiment 1 provide support for the contention that deprivation state at the time of initial exposure to a specific food substance will influence the incentive value of that substance. Subjects initially exposed to the reinforcer under a relatively high-deprivation state later responded as if they were being conditioned and extinguished under a relatively high-incentive condition. This finding seems directly applicable to the residual deprivation effect obtained in instrumental conditioning (Capaldi, 1971, 1972a; Capaldi \& Hovancik, 1973; Zaretsky, 1966). In these studies, subjects trained (and first exposed to the reinforcer) under high deprivation and subsequently shifted to a lower deprivation level continued to respond faster than did subjects trained (and initially exposed to the reinforcer) under the low deprivation state.

Experiment 2 was designed to determine whether the residual deprivation effect can be interpreted as an incentive-related phenomenon in which subjects that are shifted from high to low deprivation respond at relatively high levels because of initial exposure to the reinforcer under high deprivation. From the current viewpoint, a residual deprivation effect should occur only if the reinforcer is a novel substance (i.e., the initial exposure to the reinforcer is under high deprivation for the shifted group). In Experiment 2, two groups were runway trained under high deprivation and two groups were trained under low deprivation. In the test deprivation, or shift, phase, all groups received continued reinforcement training under low deprivation. Prior to runway training, one group in each of the high- and low-deprivation conditions received exposure to the reinforcer under low deprivation. This was performed in order to familiarize the groups with the reward prior to runway training. Because the results of Experiment 1 provided only suggestive evidence for the effect of familiarization with the reward, an extended period of familiarization training under ad-lib feeding conditions was used in Experiment 2.

\section{Method}

Subjects and Apparatus. The subjects were 32 experimentally naive, 150-day-old rats of the same description as in Experiment 1, and the apparatus was the same as in Experiment 1.

Design. A 2 by 2 factorial design was again employed, with three experimental stages. The four groups $(n=8)$ were designated as in Experiment 1: HiD-F, LoD-F, HiD-N, and LoD-N. The first stage was again a familiarization procedure. However, in the second, or conditioning, phase, subjects received acquisition training in the runway. The third stage was the test phase, in which the subjects received continued reinforcement training under low deprivation. 
Procedure. Twenty-five days prior to the start of the conditioning phase, subjects in Groups HiD-F and L.OD-F were allowed to consume 22 pellets per day in their home cages for 14 days under ad-lib feeding conditions. Subjects were fed pellets at the time corresponding to their subsequent runway training time. Over the next 11 days, the subjects were placed on a deprivation schedule to reduce their body weights to a level appropriate for the conditioning phase. The deprivation schedule was the same as the one used by Capaldi (1972a) for a fixed percentage body weight loss. High-deprivation subjects were reduced to $80 \%$ of their ad-lib body weights, while the low-deprivation subjects were reduced to $95 \%$ of their original weights. During adjustments of body weights, all subjects were handled for $5 \mathrm{~min}$ a day. All subjects were allowed to explore the apparatus for $5 \mathrm{~min}$ a day over the last 4 days, and subjects in the novel reward conditions, Groups HiD-N and LoD-N, were allowed for the first time to consume 22 pellets in their home cages for 3 days under their appropriate conditioning weight. All handling, exploration, and feeding were conducted at times corresponding to subsequent runway training times.

During the conditioning phase, each subject recejved five trials per day for 6 days. The reward was five pellets on each trial. The running procedure was the same as in the previous study, except that subjects were run in squads of eight with two subjects from each group throughout the experiment. Cage feeding followed training in the runway.

Following the preshift period, it took 10 days to adjust all high-deprivation subjects to $95 \%$ of their original body weights. During the first 3 days of readjustment, subjects in the familiar reward conditions (Groups HiD-F and LoD-F) received 22 pellets in their home cages. In the test phase, all subjects received five reinforced trials per day for $\mathbf{4}$ days. The procedure was the same as in preshift, except that all subjects were now under low deprivation.

\section{Results}

While the data for the three response measures yielded similar information, the goal measure was statistically the most reliable. The results of the goal section are plotted as a function of blocks of five trials in the lower half of Figure 1. As may be seen in Figure 1, subjects trained under high deprivation ran faster in preshift than did low-deprivation subjects. Analysis of variance of the data over Blocks 1 and 2 and over Blocks 3 and 4 yielded a significant deprivation effect $[\mathrm{Fs}(1,28)=4.20,5.45, \mathrm{p}<.05]$.

To analyze the effect of shifting the deprivation level, a 2 by 2 by 2 analysis of variance was performed on the last 10 trials of preshift (Blocks 5 and 6) and on the first 10 trials of postshift (Blocks 7 and 8). A significant Blocks by Deprivation by Familiarity with the Reward interaction was obtained $[F(1,28)=12.41, p<.01]$. To further analyze the triple interaction in the goal section, the simple interaction effects were examined. Analysis of the data revealed a significant Deprivation by Blocks interaction for the familiar reward conditions $[F(1,28)=12.77, p<$ $.01]$. This was the result of a rapid drop in running speeds for the HiD-F group when the deprivation states were equated. An examination of the simple main effects of deprivation yielded a significant difference between HiD-F and LoD-F groups during preshift $[F(1,28)=4.17, p<.05]$, but the difference between the two groups was not significant on the first 10 trials of postshift $(F<1)$. The simple Blocks by Deprivation interaction was not significant for the novel reward conditions $[F(1,28)=1.96]$. To further examine the novel reward conditions, a separate 2 by 4 analysis of variance was performed on the novel reward groups over the last 4 days of postshift. Only the deprivation effect was significant $[F(1,14)=4.77$, $\mathrm{p}<.05$ ], which indicated that Group HiD-N was running faster than Group LoD-N during the test phase.

\section{Discussion}

The present results for the novel groups are consistent with previous findings (Capaldi, 1971, 1972a; Capaldi \& Hovancik, 1973; Zaretsky, 1966) in that subjects originally trained under high deprivation subsequently displayed higher performance levels under low deprivation than did subjects trained and maintained on low deprivation. In the novel conditions, each subjects was first exposed to the reinforcer substance at the deprivation level employed during the preshift period of training, the typical procedure in previous deprivation shift experiments. However, when the typical deprivation shift procedures were preceded by a period in which all subjects were exposed to the reinforcer under low deprivation (familiar conditions), no residual deprivation effect was obtained.

Our view is that the residual deprivation effect displayed by the novel groups occurs because the incentive level for the shifted group during postshift is higher than it is for the unshifted group. The incentive level is higher in the shifted group because their first exposure to the reinforcer was under high deprivation. Presumably, the incentive value of a reinforcer substance is altered by the deprivation state of the organism at the time of initial exposure. Within this framework, initial exposure to the reinforcer under a common deprivation level, as in the familiar conditions, would set the incentive at comparable levels for subjects subsequently trained under high or low deprivation, precluding a residual deprivation effect.

\section{GENERAL DISCUSSION}

We conclude that the incentive value resulting from the use of a given amount of a particular food reward will vary directly with the deprivation level at the time the organism is first exposed to that substance. This view provides an explanation of the residual deprivation effect. It is important to note that our framework also appears to provide a reasonable account of failures to obtain the effect in at least two respects. First, no residual effect would be expected if the reinforcer is a substance to which all subjects were routinely exposed prior to any manipulations of deprivation states. The data of Experiment 2 (the familiar reward groups) are con- 
sistent with this expectation, as are data reported by Mollenauer (1971). Second, deprivation-induced differences in the incentive value generated by a reinforcer could be expected to produce behavioral differences only when a reinforcer is present. When experimental extinction is introduced concurrent with a deprivation shift, no residual deprivation effect should occur, an implication consistent with several, if not all, findings (cf. Leach, 1971). Note that the absence of a residual effect in extinction is attributed to the absence of an incentive-generating reinforcer, not a decrease in reward accompanying the drive shift. The present framework is consistent with the finding of a residual effect of deprivation attending an incomplete reward reduction (Capaldi, Smith, \& White, 1977).

While a comprehensive comparison of the various theoretical approaches to the residual deprivation effect is beyond the scope of this paper, it is worthwhile to note both similarities and differences between the present approach and that developed by Capaldi and her associates (Capaldi, 1972b; Hovancik, 1978). Although the Capaldi formulation is habit-based, while the current approach is incentive-based, the significance of this distinction depends upon the broader theoretical consideration of how incentives and habits affect behavior. Since we assume that the ultimate relationship between incentive and behavior is associative in character (cf. McHose \& Moore, 1976a), associative vs. motivational explanatory distinctions are irrelevant. The central difference between the two approaches appears to reside in the conditions assumed necessary and sufficient for highdeprivation subjects to eventually acquire more associative strength for the instrumental response than do low-deprivation subjects. The current approach requires deprivation differences at the time of initial exposure to the food substance, while the habit-based formulation requires deprivation differences at the time of instrumental training. In this respect, the present data favor our approach, but some data (Hovancik, 1978) favor the habit-based formulation. This apparent discrepancy is difficult to intercept because the present experiment and the preponderance of previous deprivation experiments differ from the Hovancik (1978) study along several dimensions (e.g., the nature of the response requirements, presence or absence of discriminative stimuli, and the distribution of training trials).

\section{REFERENCES}

Best, M. R. Conditioned and latent inhibition in taste-aversion learning: Clarifying the role of learned safety. Journal of Experimental Psychology: Animal Behavior Processes, 1975, 104, 97-113.
Bindra, D. A unified account of classical conditioning and operant training. In W. F. Prokasy \& A. H. Black (Eds.), Classical conditioning II; Current theory and research. New York: Appleton-Century-Crofts, 1972.

Capaldi, E. D. Simultaneous shifts in reward magnitude and level of food deprivation. Psychonomic Science, 1971, 23, 357-359.

Capaldi, E. D. Effects on rats' straight alley performance of shifts in body weight as a function of method of weight maintenance. Psychonomic Science, 1972, 28, 44-46. (a)

CAPAldi, E. D. Resistance to extinction in rats as a function of deprivation level and schedule of reward in acquisition. Journal of Comparative and Physiological Psychology, 1972, 79. 90-98. (b)

Capaldi, E. D., \& Hovancik, J. R. Effects of previous body weight level on rats' straight alley performance. Journal of Experimental Psychology, 1973, 97, 93-97.

Capaldi, E. D., Smith, N. S., \& White, L. A. Control of reward expectancies by drive stimuli. Journal of Experimental Psychology: Animal Behavior Processes, 1977, 3, 178-188.

Hovancik, J. R. The effect of deprivation level during noncontingent pairings and instrumental learning on subsequent instrumental performance. Learning and Motivation, 1978, 9, $1-15$.

Hull, C. L. Principles of behavior. New York: Appleton-CenturyCrofts, 1943.

KalAT, J. W. Taste salience depends on novelty, not concentration, in taste-aversion learning in the rat. Journal of Comparative and Physiological Psychology, 1974, 86, 47-50.

Kurtz, K. H., \& Jarka, R. G. Position preference based on differential food privation. Journal of Comparative and Physiological Psychology, 1968, 66, 518-521.

LFACH, D. A. Rats' extinction performance as a function of deprivation level during training and partial reinforcement. Journal of Comparative and Physiological Psychology, 1971, 75, 317-323.

Mackintosh, N. J. The psychology of animal learning. London: Academic Press, 1974.

McHose, J. H., \& Moore, J. N. Expectancy, salience, and habit: A noncontextual interpretation of the effects of changes in the conditions of reinforcement on simple instrumental responses. Psychological Review, 1976, 83, 292-307. (a)

McHose, J. H., \& Moore, J. N. Reinforcer magnitude and instrumental performance in the rat. Bulletin of the Psychonomic Society, 1976, 8, 416-418. (b)

Mollenauer, S. O. Repeated variation in deprivation level: Different effects depending on amount of training. Journal of Comparative and Physiological Psychology, 1971, 77, 318-322.

REvusky, S. H. Hunger level during food consumption: Effects on subsequent preference. Psychonomic Science, 1967, 7, 109 110.

REvusky, S. H. Effects of thirst level during consumption of flavored water on subsequent preference. Journal of Comparative and Physiological Psychology, 1968, 66, 777-779.

Revusky, S. H. Retention of a learned increase in the preference for a flavored solution. Behavioral Biology, 1974, 11, 121-125.

Revusky, S. H., \& GARCIA, J. Learned associations over long delays. In G. H. Bower (Ed.), The psychology of learning and motivation (Vol. 4). New York: Academic Press, 1970.

Spence, K. W. Behavior theory and conditioning. New Haven, Conn: Yale University Press, 1956.

ZARETSKY, H. H. Learning and performance in the runway as a function of the shift in drive and incentive. Journal of Comparative and Physiological Psychology, 1966, 62, 218-221.

(Received for publication January 14, 1980; revision accepted June 12,1980 .) 